\title{
CARACTERÍSTICAS FÍSICO-QUÍMICAS, NUTRICIONAIS E FUNCIONAIS DA PIPOCA DE AMARANTO (AMARANTHUS SPP., AMARANTHACEAE)
}

\section{PHYSICOCHEMICAL, NUTRITIONAL AND FUNCTIONAL CHARACTERISTICS OF AMARANTO POPCORN (AMARANTHUS SPP., AMARANTHACEAE)}

\author{
Dandara Bindermann", Luana Cardoso Teixeira', Mateus Reis Santos', \\ Stefaniy Obrzut Maciel ${ }^{1}$ e María Eugenia Balbi²
}

1 - Discentes do curso de Farmácia da Universidade Federal do Paraná;

2 - Docente no curso de Farmácia da Universidade Federal do Paraná, Laboratório de Bromatologia, Curitiba. Autor para correspondência: bromatologia.ufpr@gmail.com

\section{RESUMO:}

O amaranto é um pseudocereal com elevado valor nutricional, podendo ser preparado de várias maneiras e apresentando várias propriedades funcionais, como, elevada quantidade de proteínas, gorduras e minerais (cálcio, ferro, fósforo e magnésio) e boa quantidade de fibras alimentares. O objetivo deste estudo é analisar os valores nutricionais e propriedades funcionais da pipoca de amaranto com a pipoca de milho e o grão de amaranto (ambos por meio de dados presentes na literatura), e difundir o consumo do amaranto na alimentação brasileira. Após expandir o grão de amaranto, foram utilizados métodos para determinar as propriedades da pipoca de amaranto. Os resultados obtidos foram satisfatórios, com base na literatura, sendo que os dados para a pipoca de amaranto ainda são escassos, devido este ser um produto relativamente novo. O produto pode ser uma alternativa para celíacos, visto que a pipoca de amaranto não contém glúten e contém uma boa quantidade de minerais (a maioria dos produtos sem glúten são limitantes em minerais).

Palavras-chave: Amaranto; Pipoca de Amaranto; Composição Nutricional; Pseudocereal.

\begin{abstract}
:
Amaranth is a pseudocereal with high nutritional value, it can be prepared in various ways and has several functional properties, such as high protein, fat and minerals (calcium, iron, phosphorus and magnesium) and good amount of dietary fibers. The aim of this study is to analyze the nutritional values and functional properties of amaranth popcorn with corn popcorn and amaranth grain (both through literature data), and to disseminate the consumption of amaranth in the Brazilian diet. After expanding the amaranth grain, methods were used to determine the properties of amaranth popcorn. The results obtained were satisfactory, based on the literature, being that the data for amaranth popcorn are still scarce due to the fact that it is a relatively new product. The product may be an alternative for celiacs, as amaranth popcorn is gluten free and contains a good amount of minerals (most gluten free products are mineral limiting).
\end{abstract}

Keywords: Amaranth; Amaranth Popcorn; Nutritional Composition; Pseudocereal.

\section{INTRODUÇÃO}

O amaranto, pertencente ao gênero botânico Amaranthus, trata-se de um pseudocereal da classe das dicotiledôneas, da família das amarantáceas (NATIONAL 
RESEARCH COUNCIL,1989). Acredita-se que o mesmo tenha se originado na América Central (TEUTONICO; KNORR,1985); sendo seu nome advindo de duas palavras gregas que significam "imortal” ou "não perecível" (SAUNDERS; BECKER,1984).

Embora pouco estudado, há mais de 60 espécies já identificadas do gênero Amaranthus; sendo a Amaranthus cruentus L., Amaranthus caudatus L. e Amaranthus hypocondriacus L. (BREENE,1991) as mais consumidas e estudadas mundo afora.

Todas as espécies possuem características morfológicas semelhantes, de sementes pequenas e em forma lenticular, com coloração variando do branco, ao bege, bege claro, marrom e preto. A planta em si, quando ereta, pode alcançar até dois metros de altura (SAUNDERS; BECKER,1984).

Sabe-se que os grãos de amaranto constituíram a base alimentar de diversas civilizações pré-colombianas, como os maias, incas e astecas. Nessas civilizações, o amaranto era consumido diariamente e considerado sagrado, sendo utilizado em rituais religiosos - os grãos eram amassados com sangue humano e oferecido aos deuses -. 0 amaranto caiu em desuso, sendo praticamente banido, no período colonial, com a chegada dos espanhóis à região e posterior pressão dos mesmos para a erradicação de cerimônias pagãs; assim, o cultivo de amaranto acabou sendo esquecido, mantendo-se espalhado em poucas regiões andinas (BREENE,1991; BRESSANI,1989b; KALINOWSK,1993; YÁNES et al., 1994).

No Brasil, os esforços para aumentar o conhecimento da população sobre o amaranto, seu cultivo e consumo são recentes e se devem, em sua maior parte, a seu elevado valor nutricional, versatilidade de preparo e propriedades funcionais. Alguns nomes populares da planta são: Caruru, Caruru-bravo, Bredo, Caruru-verde, entre outros (TEUTONICO; KNORR, 1985).

Diferente dos demais cereais e leguminosas, o amaranto é capaz de se desenvolver em condições desfavoráveis, com luminosidade intensa, altas temperaturas e restrição hídrica (AMAYA-FARFAN et al., 2005); tal característica confere ao mesmo a possibilidade de ser cultivado em regiões áridas, sendo uma alternativa para alimentação de comunidades rurais com dificuldades financeiras.

Os grãos de amaranto apresentam uma elevada quantidade de proteínas, gorduras e minerais, principalmente quando comparado aos cereais. A sua composição proteica é considerada de alta qualidade biológica, correspondente ao seu conteúdo em lisina (geralmente limitante nos demais grãos) e outros aminoácidos essenciais (TEUTONICO; KNORR, 1985). Além disso, sabe-se que o mesmo possui uma boa quantidade de fibras 
alimentares, além de ser rico em minerais como o cálcio, ferro, fósforo e magnésio; no entanto, é pobre em vitaminas.

Inclusive, há diversos estudos que visam explorar a incorporação do grão em receitas e produtos alimentícios para crianças, pôr o mesmo apresentar alta densidade calórica e baixa viscosidade. Na África e Índia, os grãos e as folhas do amaranto vêm sendo utilizados como alimentos de baixo-custo em suplementos alimentares para recémnascidos e crianças, evitando a desnutrição (FERREIRA, T. A. P. C., et al., 2007).

Os grãos podem ser consumidos de diversas formas (BREENE, 1991), como na salada, cozido (em substituição ao arroz) ou até mesmo expandido (como pipoca), forma física que será analisada neste trabalho.

O pipocamento dos grãos é a maneira mais fácil de processar os mesmos, além de obter-se um produto final de sabor agradável (BRESSANI et al.,2006).

Sabe-se que os métodos de processamento que envolvem essa expansão do grão não danificam o anel diplóide do embrião do grão e, se o fazem, é de forma branda; isso contribui para a preservação do valor nutritivo do grão, bem como sua qualidade proteica e a atividade das vitaminas lipossolúveis (LEHMANN, 1992 apud LEHMANN, 1996).

O mecanismo de expansão do grão foi descrito por Tovar et. al (1994) e por Berghofer \& Schoenlechner (2002) e se assemelha ao mecanismo da pipoca de milho. Durante o aquecimento dos grãos, ocorre mudança do estado de água do grão, de líquido para vapor, em um pequeno intervalo de tempo, promovendo a expansão do grão. Esse vapor interno cozinha diferentes tecidos do grão, causando a expansão de grânulos de amido, a desnaturação de proteínas e ruptura das cascas.

Ainda segundo Tovar et. al (1994), a qualidade do pipocamento do grão irá variar com sua espécie e o teor de umidade dos mesmos. Segundo estudo de Bianchini \& Beléia (2010), o qual buscou analisar a influência do teor de umidade e da temperatura de armazenamento dos grãos com a capacidade de expansão térmica, o congelamento dos grãos favorece o pipocamento do mesmo e a produção de pipocas de amaranto pelo método de chapa aquecida reduz os teores de proteínas e fibras insolúveis e aumenta os de lipídeos e fibras solúveis, em comparação aos grãos crus.

Assim, tendo em vista as informações relatadas, o objetivo deste trabalho foi comparar os valores nutricionais e propriedades funcionais do grão cru da pipoca de amaranto e, ainda, da pipoca de milho (por meio de dados presentes na literatura), comparar o custo-benefício do grão, analisando se este poderia ser um potencial substituto para outros grãos, e difundir o consumo do amaranto na alimentação brasileira, além de 
estudar a criação de um possível produto alternativo para suprir a deficiência em minerais que muitos celíacos apresentam, visto que o amaranto é destituído de glúten e rico em minerais.

\section{MATERIAL E MÉTODOS}

As amostras foram avaliadas quanto à composição e determinada com base nos métodos físico-químicos para análise de alimentos descritos no Instituto Adolf Lutz, 2008 (IAL, 2008). Todas as análises foram conduzidas no laboratório de Bromatologia, localizado no Setor de Ciências da Saúde, na Universidade Federal Do Paraná. As análises foram realizadas em triplicata.

\subsection{AMOSTRA}

Os grãos de amaranto obtidos são sementes pequenas e de forma lenticular, com coloração variando do branco, ao bege, bege claro (em maior quantidade), marrom e preto. Foram adquiridos $0,406 \mathrm{~kg}$ de amaranto em uma casa de grãos em Curitiba, localizada no endereço: Rua Mariano Torres, 948 - Centro, Curitiba, PR, 80060-120.

Foi separada cerca de $0,203 \mathrm{~kg}$, cerca da metade da quantidade de grãos obtida, para realizar a expansão dos mesmos. Os grãos foram pipocados à $100^{\circ} \mathrm{C}$ em uma chapa de aquecimento de laboratório e posteriormente analisados.

\subsection{DETERMINAÇÕES ANALÍTICAS}

- Determinação da porção: A determinação da porção foi realizada através dos métodos de cálculos da ANVISA (BRASIL, 2005). A medida caseira utilizada foi de uma xícara de chá, segundo a RDC n²59 (BRASIL, 2002).

- Determinação da umidade (I.A.L,2008).

- Determinação de proteínas: Para a determinação das proteínas, foi utilizado o método de nitrogênio total (AOAC, 1995) através da técnica de Micro-Kjeldahl. A conversão para proteína bruta foi feita através do fator de conversão para proteína de origem vegetal 5,75, segundo RDC no 360 (BRASIL,2003).

- Determinação de lipídeos (IAL, 2008).

- Determinação de fibras (AOAC, 1970). 
- Determinação de resíduo mineral (IAL, 2008).

- Determinação de cálcio: A determinação dos valores de Cálcio presentes na amostra foi realizado pela titulação da amostra com EDTA (IAL, 2008).

- Determinação de carboidratos: A determinação de carboidratos foi realizada pelo método do NiFEXT, obtidos por diferença, segundo a RDC nำ60 (BRASIL,2003).

\section{RESULTADOS E DISCUSSÃO}

Os resultados das análises nutricionais da pipoca de amaranto estão apresentados na Tabela 1, da Composição Química e Nutricional da Pipoca de Amaranto em 100 gramas de produto, em base úmida.

Quanto aos valores obtidos de umidade da pipoca do amaranto (Tabela 1), foi possível observar uma grande diminuição se comparados aos valores do grão do amaranto, sendo que os nossos resultados obtidos foram de 2,00 $\pm 0,06$ enquanto que os valores obtidos por Amaya-Farfan et al. (2003) para o grão foi entre 9,2 e 13,7\%. A pipoca de amaranto também fica para trás quando comparada com a pipoca de milho, comumente consumida pela população, onde o conteúdo de umidade está entre 9,42 e 12,17\% (Nascimento \& Bouteux, 1994).

Proteínas são constituídas por subunidades denominadas aminoácidos. Oito deles podem ser sintetizados pelo corpo, sendo denominados aminoácidos essenciais. Outra classificação desses aminoácidos é dada como não-essenciais, ou seja, são fontes de proteínas corporais, encontradas no plasma, tecido visceral e músculo. Toda essa proteína faz parte das estruturas teciduais ou existe na forma de importantes componentes dos sistemas metabólicos e hormonais (MARANGON, 2004). Sementes de amaranto têm um alto valor de componentes bioativos com importantes propriedades nutricionais e nutracêuticas positivas para a saúde humana, como proteínas, aminoácidos essenciais, lipídios entre outros. A qualidade proteica e de aminoácidos essenciais da semente amaranto é superior, quando comparada a outros cereais (ORONA-TAMAYO, 2016). A quantidade de proteínas presentes nas sementes de amaranto varia, segundo Venskutonis, 2013, de 12,5 a 16\% e de 12,6 a 14,6\% de acordo com Yánes et al., 1994, dependendo da espécie em estudo. $O$ resultado encontrado da concentração proteica presente na amostra da pipoca de amaranto foi de 13,07\%, sendo que, segundo GONZÁLEZ, 2002, a semente crua possui uma média de $15 \%$ de proteína. Esse valor proteico menor obtido pelo presente estudo pode ser justificado pelo fato de que o processo de expansão do grão cru está 
relacionado à diminuição do teor de proteínas e fibras insolúveis na pipoca (BIANCHINI, 2010).

Os lipídios são considerados fontes importantes de energia metabólica, sendo compostos mais energéticos que as proteínas e os carboidratos (FARKAS et al., 1977). Também desempenham importantes funções em processos fisiológicos, mantendo a permeabilidade e flexibilidade das membranas celulares (CHO et al., 1985; STICKNEY \& HARDY, 1989). Segundo Botham \& Mayes, 2012 os lipídios podem ser classificados em principalmente como simples, complexos, os precursores e derivados. A pipoca de amaranto demonstrou-se mais pobre deste macronutriente em comparação com a pipoca de milho, apresentando aproximadamente 3 vezes menos lipídios. Observando a tabela 4, o grão de amaranto ao ser pipocado, perde uma pequena quantidade de lipídios, isso pode ser devido ao método utilizado para a determinação de lipídios - método de Soxhlet - este método exige um grande tempo para a extração, o que pode acarretar que, em amostras que possuem elementos termolábeis, podem sofrer decomposição térmica. Porém, o grão de amaranto possui maior teor de lipídios do que a quinoa e a maioria dos grãos de cereais, apresentando conteúdo de lipídios entre 5,7\% e 10,9\% (média de 8,81\%) (ALVAREZJUBETE, ARENDT, GALLAGHER, 2009; SCHOENLECHNER, SIEBENHANDL, BERGHOFER, 2008). Sendo assim, o baixo teor de lipídios pode ser uma característica satisfatória, visto que, a riqueza em gordura pode influenciar no armazenamento de algumas substâncias, uma vez que a gordura é bastante instável, podendo rancificar facilmente; os alimentos rancificados, por conseguinte, perdem determinados nutrientes, como vitaminas lipossolúveis e alguns ácidos graxos.

As fibras alimentares, do ponto de vista da nutrição humana, o termo tem sido redefinido como polissacarídeos de plantas e lignina não sujeitos à hidrólise das enzimas humanas no intestino delgado (BOUSQUET-SANTOS, TORRES, 2014). Ela é constituída de polímeros de carboidratos, com três ou mais unidades monoméricas, e mais a lignina um polímero de fenilpropano, e podem ser divididas em solúveis e insolúveis. Os efeitos positivos da fibra alimentar estão relacionados, em parte, ao fato de que uma parcela da fermentação de seus componentes ocorre no intestino grosso, o que produz impacto sobre a velocidade do trânsito intestinal, sobre o $\mathrm{pH}$ do cólon e sobre a produção de subprodutos com importante função fisiológica (BERNAUD, RODRIGUES, 2013). Saunders \& Becker (1984), constataram que o conteúdo percentual de fibra, assim como o de proteína e gordura, do amaranto eram superiores ao dos cereais comuns, demonstrando uma vantagem do amaranto, já que um alto conteúdo de fibras é bom considerando os efeitos 
benéficos destas. Após determinação de fibras, foi possível observar que, em comparação com a pipoca de milho, esta possui cerca de $2,1 \%$ a mais de conteúdo percentual de fibras em relação à pipoca de amaranto, dado apresentado na Tabela 3. Porém, este dado isoladamente não nos diz que a pipoca de milho seria melhor, afinal devemos levar em conta os outros componentes dela, como por exemplo o alto teor de lipídeos que não é muito vantajoso, especialmente para pessoas que procuram uma alimentação menos calórica.

Os sais minerais, embora menos mencionados do que os mencionados acima, são nutrientes de extrema importância para o organismo humano; neste, apresentam função plástica e reguladora, contribuindo para diversas funções metabólicas (ÁVILA). Dos 28 minerais existentes, 12 são essenciais e podem ser divididos em dois grupos de acordo com a necessidade diária: macrominerais, quando a necessidade diária é maior que 100mg, estando ligados à estrutura e formação dos ossos, regulação dos fluidos corporais e secreções digestivas, como o cálcio e o sódio; e em microminerais, quando a necessidade diária é inferior a $100 \mathrm{mg}$, estando relacionados com reações bioquímicas, ao sistema imunológico e ação antioxidante, como o ferro e o zinco (ÁVILA).

$O$ teor destes nos alimentos pode ser determinados através da determinação do resíduo mineral fixo (I.A.L,2008), método que se baseia na análise da quantidade de cinza produzida após aquecimento da amostra à temperatura de 500 a $600^{\circ} \mathrm{C}$ por menos de quatro horas, visto que o teor de cinza pode permitir, na maioria das vezes, uma estimativa do teor em cálcio e fósforo do alimento analisado. Entretanto, para produtos vegetais, como o amaranto analisado neste artigo, a técnica pode apresentar muitas variações quanto aos valores estimados. Na presente análise, obteve-se um teor de cinzas na pipoca de amaranto maior quando comparada ao grão in natura.

Sabe-se, através da literatura, que o amaranto é rico em minerais como o cálcio, magnésio, fósforo e zinco; além de possuir um menor teor de ácido fítico, taninos e oxalatos, o que permite uma melhor absorção do cálcio no organismo. Através da determinação do resíduo fixo para cálcio (I.A.L,2008), obteve-se 40,04mg para 100 gramas de produto, um valor considerado baixo quando comparado ao teor de cálcio no grão de amaranto in natura, a qual apresenta cerca de 180,1-217mg de cálcio para 100 gramas de produto (ALVAREZ, J. et al). Tal redução no teor de cálcio pode ter ocorrido pela extrusão do grão ao produzir a pipoca de amaranto ou, então, por características intrínsecas - referente a variedade, época de colheita, armazenamento, etc - dos grãos utilizados para determinação.

Para quantificar os valores de carboidratos presentes na amostra foi utilizado 0 
método do NiFEXT, obtidos por diferença, segundo a RDC nํㅜ잉. Observamos um valor de 75,95 gramas de carboidrato para $100 \mathrm{~g}$ de amostra, valor o qual está muito perto do valor previsto para o grão de amaranto; segundo estudos do Ministério da Agricultura, Pecuária e desenvolvimento, o teor de carboidratos neste equivale à $72,5 \mathrm{~g} \mathrm{em} 100 \mathrm{~g}$ de amostra. Quando colocamos de frente os valores da pipoca de amaranto com a pipoca do milho, notamos uma diferença entre os valores, uma vez que para $100 \mathrm{~g}$ de pipoca de milho tem-se $61,29 \mathrm{~g}$ de carboidrato (TISSOT, ZAMBIASI \& MENDONÇA).

$\mathrm{Na}$ Tabela 2, da Informação Nutricional da pipoca de amaranto, têm-se os teores nutricionais na porção de 44 gramas do produto. Nesta, pode-se destacar a alta porcentagem dos para os valores diários de referência dos carboidratos e fibras.Tais valores permitem ressaltar o quão rico em nutrientes pode ser a pipoca de amaranto, podendo suprir necessidades nutricionais mesmo que consumida em pouca quantidade.

Tabela 1. Resultados da composição química e nutricional da Pipoca de Amaranto (Amaranthus spp., Amaranthaceae) em 100g.

\title{
Determinação
}

$g / 100 g$

Umidade

$2,00 \pm 0,06$

Proteína ${ }^{1}$

$13,07 \pm 0,06$

Lipídios

$5,13 \pm 0,64$

Carboidratos $^{2}$

75,95

Fibras

$12,2 \pm 1,83$

Minerais

$2,78 \pm 0,022$

Cálcio

$0,04004 \pm 0,06$

\begin{abstract}
1 Usou-se o fator 5,75 para conversão em proteína, segundo as recomendações da RDC 360 (BRASIL, 2003)

2 Obtido por diferença
\end{abstract}


Tabela 2. Informação Nutricional ${ }^{*}$ da Pipoca de Amaranto (Amaranthus spp., Amaranthaceae) na medida caseira que corresponde a $44 \mathrm{~g}$, uma xícara de chá.

\begin{tabular}{ccc}
\hline Informações & 1 Xícara de chá (44g) & VD\%* \\
\hline Valor calórico & 187,26 & 9,36 \\
Carboidratos & 36,16 & 25,32 \\
Proteínas & 5,72 & 7,63 \\
Gorduras totais & 2,26 & 4,11 \\
Fibras & 5,37 & 21,5 \\
Cálcio & 0,0176 & 1,76
\end{tabular}

* Valores diários de referência com base em uma dieta de $2.000 \mathrm{Kcal}$ ou $8.400 \mathrm{~kJ}$, segundo as recomendações da RDC 360 (BRASIL, 2003)

Tabela 3. Comparação da composição química e nutricional da Pipoca de Amaranto (Amaranthus spp., Amaranthaceae) em 100g, com outros grãos.

\begin{tabular}{ccc}
\hline Determinação & Pipoca de Amaranto & ${\text { Pipoca de } \text { Milho }^{2}}^{\text {Umidade }}$ \\
Proteína & 2,00 & 2,80 \\
Lipídios & 13,0 & 9,90 \\
Carboidratos & 5,13 & 15,9 \\
Fibras & 75,95 & 70,3 \\
Cálcio & 12,2 & 14,3 \\
Kcal & 0,0176 & 3 \\
\end{tabular}

1 Valores centesimais segundo a Tabela Brasileira de Composição de Alimentos TACO, 4a edição

2 Pipoca de milho com óleo de soja, sem sal 
Tabela 4. Comparação da composição química e nutricional da Pipoca de Amaranto (Amaranthus spp., Amaranthaceae) e do Grão de Amaranto.

\begin{tabular}{ccc}
\hline Determinação & Pipoca de Amaranto (\%) & Grão de Amaranto ${ }^{\mathbf{1}}(\%)$ \\
\hline Umidade & 2,00 & 11,3 \\
Proteína & 13,0 & 13,6 \\
Lipídios & 5,13 & 7,02 \\
Carboidratos & 75,95 & 65,3 \\
Fibras & 12,2 & 6,70 \\
Cálcio & 0,0176 & 159 \\
Kcal & 402,25 & 365
\end{tabular}

\section{Valores centesimais segundo a Tabela Brasileira de Composição de Alimentos - TBCA, versão 6 de 2017}

\section{CONCLUSÃO}

Com base nos resultados obtidos através das determinações realizadas, pode-se concluir que o amaranto poderia ser realmente uma possibilidade de substituição de outros grãos, como o arroz e o feijão. Pode-se evidenciar que a pipoca de amaranto manteve as características nutricionais do grão de amaranto, sendo rica em fibras, proteínas e não apresentando um alto teor de lipídeos. Quanto ao teor de cálcio, notou-se uma perda significativa do mineral quando comparada ao grão in natura; entretanto, outras determinações e estudos podem ser realizados, a fim de avaliar se este poderia ser utilizado, ou não, para suplementar a deficiência de minerais que muitos celíacos apresentam. Tal grão ainda constitui uma alternativa de alimento sem glúten para intolerantes ou indivíduos que optam por uma dieta gluten-free, visto que, apesar de o milho também ser isento de glúten, a pipoca de amaranto analisada proporciona ainda outras qualidades nutricionais, tornando-a uma ótima opção para se acrescentar na dieta alimentar. 


\section{REFERÊNCIAS}

BRASIL, Agência de Vigilância Sanitária (ANVISA). Resolução da diretoria colegiada- RDC no 259, de 20 de setembro de 2002. Disponível em: <http://portal.anvisa.gov.br/legislacao\# /visualizar/26993> Acesso em: 03 set. 2019.

BRASIL, Agência de Vigilância Sanitária (ANVISA). Resolução da diretoria colegiada- RDC no 360, de 23 de dezembro de 2003. Disponível em: <http://portal.anvisa.gov.br/documents/ 33880/2568070/res0360_23_12_2003.pdf/5d4fc713-9c66-4512-b3c1-afee57e7d9bc> Acesso em: 03 set. 2019.

ALMEIDA, Simone Gonçalves; CORRÊA DE SÁ, Wildemara Almeida. Amaranto (amaranthus spp) e quinoa (chenopodium quinoa) alimentos alternativos para doentes celíacos. Ensaios e ciência: ciências biológicas, agrárias e da saúde, Campo Grande, ano 2009, v. XIII, n. 1, p. 77-92, 2009. Disponível em: http://www.redalyc.org/articulo.oa?id=260 12800008. Acesso em: 6 set. 2019.

ALVAREZ-JUBETE, L; ARENDT, E.K; GALLAGHER, E. Nutritive value of pseudocereals and their increasing use as functional gluten-free ingredients. Trends in Food Sciences and Technology, v. 21, p. 106-113,2010.

AMAYA-FARFAN, Jaime; MARCÍLIO, Roberto; SPEHAR, Carlos Roberto. Deveria o Brasil investir em novos grãos para a sua alimentação? A proposta do amaranto (Amaranthus sp.). Segurança alimentar e nutricional, Campinas, 2005. Disponível em: https://periodicos.sbu.unicamp.br/ojs/index.php/san/article/view/1838/1891. Acesso em: 3 set. 2019.

AOAC INTERNATIONAL. Official methods of analysis of the AOAC International. 16 ed. Arlington: AOAC International, 1998. 1298p.

ASCHERI, José Luis Ramírez; CARVALHO, Carlos Wanderlei Pier de; SPEHAR, Carlos Roberto. A extrusão do amaranto no desenvolvimento de produtos: caracterização físicoquímica. Embrapa agroindústria de alimentos, Rio de Janeiro, dezembro 2004. Disponível em: https://www.embrapa.br/busca-de-publicacoes/-/publicacao/415867/a-extrusao-do- 
amaranto-no-desenvolvimento-de-produtos-caracterizacao-fisico-quimica. Acesso em: 3 set. 2019.

ÁVILA, M. A Importância dos Sais Minerais. Disponível em:<http://www.endocrinologia.com.br /nutricao/a-importancia-dos-sais-minerais.php>. Acesso em: 6 nov 2019.

BECKER, R.; Saunders, R. M. Amaranthus. A potential food and feed resource. Advances in, E; SCHOENLECHNER, R. Grain amaranth. In: BELTON, P.S; TAYLOR, J.R.N. Pseudocereals and less common cereals: grain properties and utilization potential. Cereal Science and Technology, v.6, p.257-396,1984.

BERGHOFER New York: Springer, 2002. p 219-260.

BERNAUD, Fernanda Sarmento Rolla; RODRIGUES, Ticiana C. Fibra alimentar: ingestão adequada e efeitos sobre a saúde do metabolismo. Arq Bras Endocrinol Metab, São Paulo, v. 57, n. 6, p. 397-405, Aug. 2013. Disponível em: <http://www.scielo.br/scielo.php?script= sci_arttext\&pid=S0004-27302013000600001\&lng=en\&nrm=iso>. Acesso em: 05 nov. 2019.

BIANCHINI, Maria das Graças de A.; BELÉIA, Adelaide Del Pinto. Umidade e congelamento dos grãos de amaranto e sua capacidade de expansão térmica. Pesquisa agropecuária brasileira, Brasília, 08 2010. Disponível em: seer.sct.embrapa.br/index.php/pab/article/view/7940/6088. Acesso em: 3 set. 2019.

BOUSQUET-SANTOS, K.; TORRES, A. Nutrição Humana. Universidade de Brasília, Brasília, 2014.

BREENE, W.M. Food uses of grain amaranth. Cereal Foods World, St. Paul, v. 36, n. 5, p. 426-429, 1991.

BRESSANI, R. Amaranth. The nutritive value and potential uses of the grain and byproducts. Food and Nutrition Bulletin, Tokyo, v. 10, n.2, p. 49-59, 1988.

CAPRILES, Vanessa Dias; COELHO, Karina Dantas; MATIAS, Andréa Carvalheiro Guerra; ARÊAS, José Alfredo Gomes. Efeito da adição de amaranto na composição e na 
aceitabilidade do biscoito tipo cookie e do pão de forma. Alim. Nutr., Araraquara, julho/setembro 2006. Disponível em: https://www.researchgate.net/publication/49599828 Efeito_da_adicao_de_amaranto_na_composicao_e_na_aceitabilidade_do_biscoito_tipo_c ookie_e_do_pao_de_forma. Acesso em: 5 set. 2019.

COELHO, Karina Dantas. Desenvolvimento e avaliação da aceitação de cereais matinais e barras de cereais à base de amaranto (Amaranthus cruentus L.). PRONUT, São Paulo, 2006. Disponível em: https://www.teses.usp.br/teses/disponiveis/89/89131/tde-25102006105736/publico/karina_mestrado_pronut.pdf. Acesso em: 30 ago. 2019.

FERREIRA, T. A. P. C.; MATIAS, A. C. G.; ARÊAS, J. A. G. Características nutricionais e funcionais do Amaranto (Amaranthus spp.). Sociedade brasileira de alimentação e nutrição, São Paulo, 08 2007. Disponível em: https://www.researchgate.net/publication/241194516_ Caracteristicas_nutricionais_e_funcionais_do_Amaranto_Amaranthusspp. Acesso em: 3 set. 2019.

GONZÁLEZ, R. J.; TORRES, R. L.; GREEF, D. M. de; TOSI, E.; RE, E. Effects of popping and extrusion processes on some hydration properties of amaranth. Brazilian Journal of Chemical Engineering, São Paulo, v. 19, ed. 4, 12 2002. Disponível em: http://www.scielo.br /scielo.php?script=sci_arttext\&pid=S0104-66322002000400006. Acesso em: 5 nov. 2019.

I.A.L, Instituto Adolfo Lutz. Normas analíticas do Instituto Adolfo Lutz: métodos químicos e físicos para análise de alimentos. 4 ed. Brasília: Anvisa, 2005. 1018p.

KALINOWSK, L. S. La Kiwicha y su cultivo, Cuadernos de Capacitación Popular, v. 45, p. 9-79, 1993.

LEHMAN, J.W. Case history of grain amaranth as an alternative crop. Cereal Foods World, v. 41, n.5, p. 399-410, 1996.

MARANGON, Antônio Felipe Corrêa; MELO, Renata Adjuto de. Consumo de proteínas e ganho de massa muscular. Universitas Ciências da Saúde, [s. I.], v. 2, ed. 2, p. 281-290, 2004. Disponível em: https://www.publicacoesacademicas.uniceub.br/cienciasaude/article/ viewFile/541/361. Acesso em: 5 nov. 2019. 
MELO, José Fernando Bibiano; NETO João Radünz; SILVA José Henrique Souza da; TROMBETTA Carlos Guilherme. Desenvolvimento E Composição Corporal De Alevinos De Jundiá (Rhamdia Quelen) Alimentados Com Dietas Contendo Diferentes Fontes De Lipídios. Ciência Rural, Santa Maria, v.32, n.2, p.323-327, 2002. Disponível em: http://www.scielo.br/pdf/\%0D/cr/v32n2/a23v32n2.pdf. Acesso em: 2 nov. 2019.

National Research Council. Amaranth: modern prospects for an ancient crop. Washington: National Academy Press,1984. 81p.

ORONA-TAMAYO, Domancar Luka; PAREDES-LOPEZ, Octavio. Amaranth Part 1Sustainable Crop forthe 21st Century: Food Properties and Nutraceuticals for Improving Human health. Sustainable Protein Sources, [s. I.], ed. 1, p. 239-256, 10 2016. Disponível em: https://www.researchgate.net/publication/306432698_Amaranth-Sustainable_crop_for _the_21st_century_food_properties_and_nutraceuticals_for_improving_human_health. Acesso em: 5 nov. 2019.

PIEROTTI, Soraia Mendes. Amaranto - análises físico-químicas e funcionais - comparação com dados presentes na literatura. Londrina, 2013. Disponível em: http://repositorio.roca.utfpr.edu.br/jspui/bitstream/1/1516/1/LD_COALM_2012_2_09.pdf. Acesso em: 2 set. 2019.

SANTANA Márcia Cristina Araújo; RODRIGUES Juliana Huback Ferraz; CAVALI Jucilene; BULCÃO Lucas Fialho de Aragão. Lipídeos: classificação e principais funções fisiológicas. REDVET. Revista Electrónica de Veterinaria, Málaga, v.18, n.8, 2017, pp. 1-14. Disponível em: https://www.redalyc.org/pdf/636/63652581012.pdf. Acesso em: 2 nov. 2019.

SAUNDERS, R. M; BECKER, R. Amaranthus: a potencial food and feed resource. Advences in Cereal Science and Technology, St. Paul, v. 6, p. 357-396,1984.

TBCA. Tabela Brasileira de Composição de Alimentos. Disponível em: <http://www.tbca.net.br /base-dados/int_composicao_alimentos.php?cod_produto=C0401A\#>. Acesso em: 06 nov 2019.

TEUTONICO, R. A.; KNORR, D. Amaranth; composition, properties and aplications of a 
rediscovered crop. Food Technology, Chicago, v. 39, n. 4, p. 49-59, 1985.

TISSOT, U. F.; ZAMBIASI, R. C.; MENDONÇA, C. R. B. Milho pipoca: caracterização física, química, microbiológica e sensorial. Boletim do centro de Pesquisa e Processamento de alimentos, v. 19, n.1, p. 1-12, 2001.

TOVAR, L.R; VALDIVIA, M.A; BRITO, E. Popping amaranth, state of the art. In: PAREDESLÓPEZ, O. (Ed). Amaranth: biology, chemistry, and technology. Boca Raton: CRC, 1994. p. 143-154.

UNIVERSIDADE DE SÃO PAULO. Faculdade de Ciências Farmacêuticas. Departamento de Alimentos e Nutrição Experimental/BRASILFOODS (1998). Tabela Brasileira de Composição de Alimentos-USP. Versão 6.0. Disponível em: http://www.tbca.net.br/ Acesso em: 2 nov. 2019.

UNIVERSIDADE ESTADUAL DE CAMPINAS - UNICAMP. Tabela brasileira de composição de alimentos - TACO. 4. ed. rev. e ampl. Campinas: UNICAMP/NEPA, 2011. Disponível em: http://www.nepa.unicamp.br/taco/contar/taco_4_edicao_ampliada_e_revisada.pdf?arquivo =taco_4_versao_ampliada_e_revisada.pdf. Acesso em: 2 nov. 2019.

VENSKUTONIS, Petras R.; KRAUJALIS, Paulius. Nutritional Components of Amaranth Seeds and Vegetables: A Review on Composition, Properties, and Uses. Comprehensive Reviews in Food Science and Food Safety, [s. I.], v. 12, ed. 4, 12 jun. 2013. Disponível em: https://onlinelibrary.wiley.com/doi/full/10.1111/1541-4337.12021. Acesso em: 5 nov. 2019.

YAMANI, Beatriz Valcárcel. Substituição parcial de farinha de trigo por farinha de amaranto (Amaranthus cruentus L.), quinoa (Chenopodium quinoa W.) e maca (Lepidium meyenii W.) na elaboração de panetone. São Paulo, 2015. Disponível em: https://www.teses.usp.br/ teses/disponiveis/9/9133/tde-14122015-170439/publico/Beatriz_Valcarcel_Yamani_DO_ corrigida.pdf. Acesso em: 2 nov. 2019. 